\title{
The CANDELLE experiment for characterization of neutron sensitivity of LiF TLDs
}

\author{
M. Le Guillou, A. Billebaud, A. Gruel, G. Kessedjian, O. Méplan, C. Destouches, P. Blaise
}

\begin{abstract}
As part of the design studies conducted at CEA for future power and research nuclear reactors, the validation of neutron and photon calculation schemes related to nuclear heating prediction are strongly dependent on the implementation of nuclear heating measurements. Such measurements are usually performed in low-power reactors, whose core dimensions are accurately known and where irradiation conditions (power, flux and temperature) are entirely controlled. Due to the very low operating power of such reactors (of the order of $100 \mathrm{~W}$ ), nuclear heating is assessed by using dosimetry techniques such as thermoluminescent dosimeters (TLDs). However, although they are highly sensitive to gamma radiation, such dosimeters are also, to a lesser extent, sensitive to neutrons. The neutron dose depends strongly on the TLD composition, typically contributing to 10 $30 \%$ of the total measured dose in a mixed neutron/gamma field. The experimental determination of the neutron correction appears therefore to be crucial to a better interpretation of doses measured in reactor with reduced uncertainties. A promising approach based on the use of two types of LiF TLDs respectively enriched with lithium-6 and lithium-7, precalibrated both in photon and neutron fields, has been recently developed at INFN (Milan, Italy) for medical purposes. The CANDELLE experiment is dedicated to the implementation of a pure neutron field "calibration" of TLDs by using the GENEPI-2 neutron source of LPSC (Grenoble, France). Those irradiation conditions allowed providing an early assessment of the neutron components of doses measured in EOLE reactor at CEA Cadarache with $10 \%$ uncertainty at $1 \sigma$.
\end{abstract}

Index Terms- nuclear heating, thermoluminescent dosimeters, neutron sensitivity

\section{INTRODUCTION}

$\mathrm{T}$ HE accurate determination of nuclear heating remains a key point in the design studies of power and research reactors. The experimental validation of neutron and photon propagation calculation schemes related to nuclear heating prediction requires the implementation of nuclear heating measurements usually performed in zero-power reactors (ZPRs). Due to the very low operating power of such reactors,

Submitted on $01 / 06 / 2017$

M. Le Guillou, A. Gruel, C. Destouches and P. Blaise are with CEA, DEN, DER, Experimental Physics Service, F-13108 Saint Paul lez Durance, France (e-mail: adrien.gruel@cea.fr).

A. Billebaud, G. Kessedjian and O. Méplan are with LPSC, Université Grenoble-Alpes, CNRS/IN2P3, 53 avenue des Martyrs, F-38026 Grenoble, France. nuclear heating is assessed by using dosimetry techniques rather than calorimetry techniques which are usually used in power reactors like MTRs. In particular, the use of thermoluminescent dosimeters (TLDs) is remarkably well suited to gamma heating. However, although they are highly sensitive to gamma radiation, TLDs are also, to a lesser extent, sensitive to neutrons. Since the latter currently relies on literature data with relatively high uncertainties (up to 50$100 \%$ ), a promising approach to experimentally determine this neutron contribution is based on the use of two types of doped lithium fluoride TLDs pre-calibrated both in gamma and neutron fields. Such approach has been recently developed for medical purposes at the national institute for nuclear physics (INFN, Milan, Italy) [1].

Within the framework of the French interdisciplinary program for nuclear, energy, environment, waste and society (NEEDS), the CANDELLE experiment is dedicated to the implementation of a pure neutron field calibration of $\mathrm{LiF}$ TLDs by using the GENEPI-2 neutron source of the laboratory of subatomic physics and cosmology (LPSC, Grenoble, France) [2]. The first phase of the project, presented in this paper, involved carrying out preliminary irradiations to ensure the exploitability of the TLD glow curves. In the next section are presented the main principles of gamma heating measurements performed at CEA Cadarache, as well as those of the discrimination method developed at INFN. In the following two sections are described the implementation and the results of the test irradiations carried out at LPSC, and a preliminary assessment of the neutron contributions to the total doses measured by LiF TLDs in the EOLE reactor.

\section{GAMMA HEATING MEASUREMENTS USING TLDS}

\section{A. Overview and general principles}

The measurement methodology with TLDs implemented at CEA Cadarache is fully described in [3], [4] and [5]-[7], and summed up hereafter. The TLD pellets are usually made of luminescent materials such as doped calcium or lithium fluorides or alumina $\left(\mathrm{CaF}_{2}, \mathrm{LiF}, \mathrm{Al}_{2} \mathrm{O}_{3}\right)$. In order to ensure the charge particle equilibrium (CPE) in the TLDs, they are encapsulated in $2 \mathrm{~mm}$ thick pillboxes made of the material to be investigated. The encapsulated TLDs are firstly calibrated in a pure gamma field nearby a ${ }^{60} \mathrm{Co}$ source, and then 
irradiated in mixed gamma-neutron field at the positions of interest in reactor. The luminescent signals emitted by the irradiated TLDs, i.e. their glow curves (GCs), are read out by thermal stimulation after withdrawal of the TLDs from the core. The absorbed doses are finally deduced from the integral of the GCs, thanks to the calibration curves and the correction factors related to the presence of the pillboxes and the neutron contribution to the total measured dose.

\section{B. Description of the gamma-neutron discrimination method}

The absorbed neutron dose strongly depends on the TLD composition, typically contributing to $10-30 \%$ of the total measured dose in mixed gamma-neutron field. Researchers from INFN recently proposed a method aimed at discriminating the gamma and neutron components of the GCs of LiF TLDs irradiated in mixed field. A complete description of this method can be found in [1], and its main principles are presented below.

As shown in Fig. 1, GCs of doped lithium fluoride TLDs (LiF:Mg,Ti) exhibit two peaks whose height ratio depends on the relative contributions of gammas and neutrons to the total absorbed dose. The method relies on the deconvolution of these two contributions from the GC of a TLD-700 $\left({ }^{7} \mathrm{Li}\right.$ enriched, i.e. low neutron sensitivity) irradiated in mixed field, using the calibration GC of the same TLD irradiated in a pure gamma field, and the one of a TLD-600 ( ${ }^{6} \mathrm{Li}$-enriched, i.e. high neutron sensitivity) irradiated with thermal neutrons.

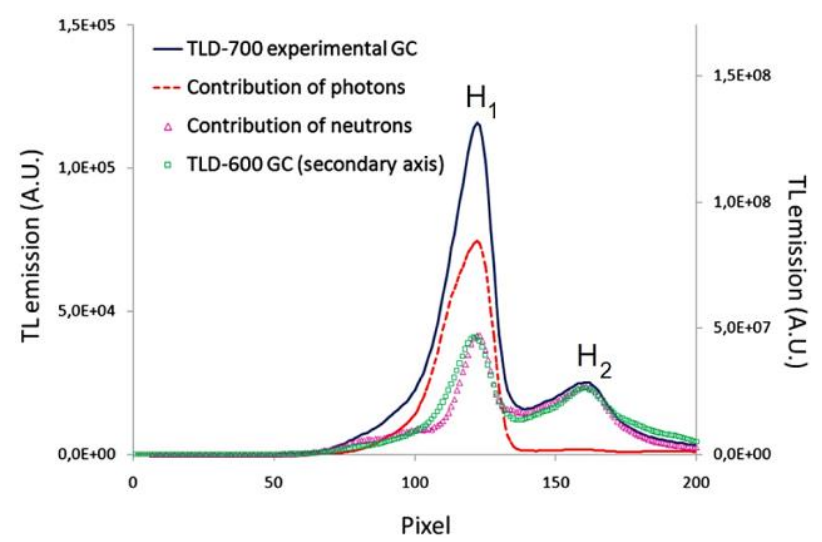

Fig. 1. Contributions of gammas (red dashes) and neutrons (pink triangles) to the GC of a TLD-700 $\left({ }^{6} \mathrm{Li} /{ }^{7} \mathrm{Li} \sim 0.01 \%\right)$ irradiated in mixed gamma-neutron field (blue line), compared with the GC of a TLD-600 $\left({ }^{6} \mathrm{Li} /{ }^{7} \mathrm{Li} \sim 95.6 \%\right)$ irradiated with thermal neutrons (green squares, secondary axis) [1].

The assumption is made that, after background subtraction, the heights $H_{1}$ and $H_{2}$ of the two peaks exhibited by the GC of a TLD-700 irradiated in mixed gamma-neutron field, i.e. in reactor, can be expressed as:

$$
\begin{aligned}
& H_{1}=D_{\gamma} H_{1}^{\gamma}+\phi_{n} H_{1}^{n}, \\
& H_{2}=D_{\gamma} H_{2}^{\gamma}+\phi_{n} H_{2}^{n},
\end{aligned}
$$

where $D_{\gamma}$ is the absolute gamma dose, $\phi_{n}$ is the thermal neutron fluence, and $H_{1}^{\gamma}, H_{1}^{n}, H_{2}^{\gamma}, H_{2}^{n}$ are the respective heights of the gamma $(\gamma)$ and neutron $(n)$ contributions to the first (subscript 1) and the second (subscript 2) peaks of the TLD-700 GC, normalized to dose and fluence units. The gamma dose in mixed field (corrected for the neutron contribution) is then given by the following relation:

$$
D_{\gamma}=\frac{H_{2} R_{n}-H_{1}}{H_{2}^{\gamma} R_{n}-H_{1}^{\gamma}} \quad \text { where } \quad R_{n}=\frac{H_{1}^{n}}{H_{2}^{n}},
$$

The $R_{n}$ ratio is obtained from the GC of an uncalibrated TLD-600 irradiated with thermal neutrons, assuming that the gamma contribution for this type of TLD is usually negligible, due to the ${ }^{6} \mathrm{Li}$ enrichment. The peak heights $H_{1}^{\gamma}$ and $H_{2}^{\gamma}$ are deduced from the TLD-700 calibration GCs in pure gamma field and normalized to dose unit. The accuracy of the method can be checked as shown in Fig. 1, where the neutron component of the TLD-700 GC (pink triangles), obtained by subtracting the gamma dose calculated with equation (3), is compared with the GC of a TLD-600 irradiated with thermal neutron (green squares).

\section{THE CANDELLE EXPERIMENT}

\section{A. Objectives}

As part of the French interdisciplinary program NEEDS, the CANDELLE experiment (French acronym for neutron calibration of enriched-lithium based luminescent detectors) aims at evaluating the feasibility of the neutron calibration of LiF TLDs by using the nuclear energy experimental platform PEREN of LPSC.

\section{B. Experimental setup}

The irradiations were carried out with the fast neutron pulsed source provided by the GENEPI-2 electrostatic accelerator of the PEREN platform [2]. Fast neutrons were produced through the D-D nuclear reaction target at an energy of $3.1 \mathrm{MeV}$.

The Li-enriched TLD square pellets $\left(3.2 \times 3.2 \mathrm{~mm}^{2}\right.$, $0.9 \mathrm{~mm}$ thick), namely TLD-600 ( $\left.{ }^{6} \mathrm{LiF}: \mathrm{Mg}, \mathrm{Ti}, 95.6 \%{ }^{6} \mathrm{Li}\right)$ and TLD-700 ( ${ }^{7} \mathrm{LiF}: \mathrm{Mg}, \mathrm{Ti}, 99.9 \%{ }^{7} \mathrm{Li}$ ), were stacked in aluminum pillboxes. The irradiation configuration is presented in Fig. 2. The pillboxes were located at around $3 \mathrm{~cm}$ from the target, under which conditions the fast neutron flux at $0^{\circ}$ was estimated to be about $5 \times 10^{3} \mathrm{n} \cdot \mathrm{cm}^{-2} \cdot \mathrm{s}^{-1}$.

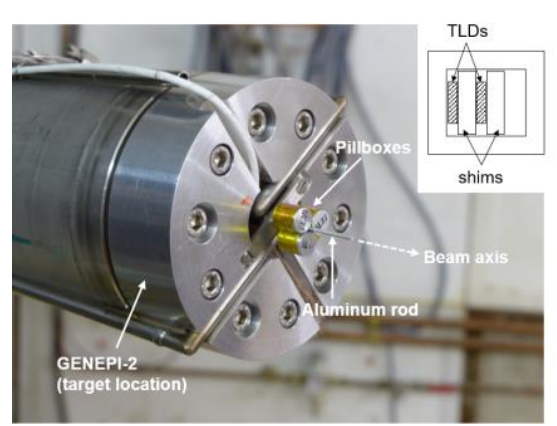

Fig. 2. Irradiation configuration of the TLDs at the PEREN platform [CNRS]. 


\section{Results and discussion}

The total fluency reached is around $2 \times 10^{8} \mathrm{n} . \mathrm{cm}^{-2}$. Considering that the gamma production due to activation of the pillbox material is negligible (preliminary calculations), the averaged peak height ratios in pure fast neutron field are $4.66 \pm 5 \%$ for TLD-600 (standard deviation of $3.7 \%$ on all TLD-600 measurements) and $4.97 \pm 5 \%$ for TLD-700 (standard deviation of $12.2 \%$ on all TLD-700 measurements).

The peak height ratios are similar for both types of TLDs. The standard deviation for TLD-700 is higher because of the very statistics compared to the one of TLD-600 (higher neutron cross-section of ${ }^{6} \mathrm{Li}$ ).

\section{EVALUATION OF THE NEUTRON CONTRIBUTIONS TO THE DOSE MEASURED IN MIXED FIELD}

\section{A. Gamma dose calibration}

TLDs were calibrated in a pure gamma field at a distance of $80 \mathrm{~cm}$ of a ${ }^{60} \mathrm{Co}$ source, whose $\beta$ - decay leads to the emission of two gamma rays at 1.17 and $1.33 \mathrm{MeV}$. TLD-600 and TLD700 were calibrated at doses ranging from $100 \mathrm{mGy}$ to $2 \mathrm{~Gy}$.

The average peak heights ratios are within 45 to $50 \pm 5 \%$. The same results are observed for TLD-600. The peak height ratios are very different from those obtained in a pure neutron flux: the second peak is almost noticeable on some GCs. Among all detectors the discrepancy of the average peak heights at different doses is quite variable as it ranges from 5 to $15 \%$.

\section{B. Irradiation in the EOLE reactor}

This analysis method has been tested on measurements carried out in the EOLE ZPR at Cadarache. Several locations in the core with different neutron and gamma spectra have been investigated with TLDs.

The order of magnitude of the fluency reached at the end of an irradiation at the core center (maximum flux) was around $5 \times 10^{10} \mathrm{n} . \mathrm{cm}^{-2}$, to be compared to the $2 \times 10^{8} \mathrm{n} . \mathrm{cm}^{-2}$ reached during the neutron calibration at the PEREN platform. Given the measurement location in the core the peak height ratios vary between 25 and 40. They indeed depend on the neutron and gamma flux shape and on the gamma/neutron ratio at the measurement location.

\section{Results and discussion}

The neutron contributions to the total doses in different locations are presented Table I. The relative uncertainty on each measurement is around 5\%. In each location several TLDs measurements were made, leading to discrepancies between 5 to $10 \%$, which is the same order of magnitude as the final uncertainty.
TABLE I

NEUTRON CONTRIBUTION IN DIFFERENT LOCATIONS OF THE CORE. THE DISCREPANCY IS THE STANDARD DEVIATION BETWEEN THE DIFFERENT TLDS MEASUREMENTS IN A SAME LOCATION

\begin{tabular}{cccc}
\hline \hline Neutron contribution & Central core & Core periphery & Water \\
\hline $\begin{array}{c}\text { Average value } \\
\text { Relative uncertainty }\end{array}$ & $9.0 \%$ & $8.7 \%$ & $11.9 \%$ \\
$(1 \sigma)$ & $5.2 \%$ & $5.2 \%$ & $5.1 \%$ \\
Relative discrepancy & $10.0 \%$ & $6.5 \%$ & $4.9 \%$ \\
\hline \hline
\end{tabular}

After the neutron calibration at the PEREN platform and the gamma calibration with a ${ }^{60} \mathrm{Co}$ source, the neutron contribution to the total dose absorbed in a mixed gammaneutron field can be assessed with a final relative uncertainty of around $10 \%$.

\section{CONCLUSION AND OUTLOOKS}

Irradiation of TLDs in a fast neutron field at the GENEPI-2 neutron source of LPSC (Grenoble, France) allowed measuring the peak height ratios for LiF TLDs, hence providing an early assessment of the neutron components of doses measured in the mixed gamma-neutron field in the EOLE reactor at CEA Cadarache with $10 \%$ uncertainty at $1 \sigma$.

\section{ACKNOWLEDGMENT}

The authors wish to acknowledge S. Rey and F. Villa from the accelerator group of LPSC, L. De Padua and N. Makaryan from the radiation protection service (SPR) of CEA Cadarache, as well as the operator team of the EOLE reactor.

\section{REFERENCES}

[1] G. Gambarini et al., "Determination of gamma dose and thermal neutron fluence in BNCT beams from the TLD-700 glow curve shape", Radiat. Meas., Vol. 45, pp.640-642, 2010. DOI: 10.1016/j.radmeas.2010.02.006.

[2] F. Villa et al., "Multipurpose applications of the accelerator-based neutron source GENEPI2". 5th International Meeting of Union for Compact Accelerator-Driven Neutron Sources (UCANS V), May 2015, Padova, Italy, Il Nuovo Cimento C 38, pp. 182, 2016. DOI: 10.1393/ncc/i2015-15182-2

[3] H. Amharrak, Ph.D. thesis, Aix-Marseille Université, France, 2012.

[4] M. Le Guillou et al., "State of the art on nuclear heating measurement methods and expected improvements in zero power research reactors", EPJ Nuclear Sci. Technol., Vol. 3, No. 11, 2017. DOI: 10.1051/epjn/2017002.

[5] H. Amharrak et al., "Analysis and recent advances in gamma heating measurements in MINERVE facility by using TLD and OSLD techniques", IEEE Trans. Nucl. Sci., Vol. 59, No. 4, pp. 1360-1368, 2012. DOI: 10.1109/ANIMMA.2011.6172901.

[6] H. Amharrak et al., "Development and optimization of nuclear heating measurement techniques in Zero Power experimental Reactors", IEEE Trans. Nucl. Sci., Vol. 61, No. 5, pp. 2515-2526, 2014. DOI: 10.1109/TNS.2014.2355917.

[7] P. Blaise et al., "Nuclear heating measurement in critical facilities and experimental validation of code and libraries - An application to prompt and delayed $\gamma$ nuclear data needs", Phys. Proc., Vol. 59, pp. 316, 2014. DOI: 10.1016/j.phpro.2014.10.002. 